\section{$\underset{\substack{\text { hommes } \\ \text { \& migrations }}}{ }$}

\section{Hommes \& migrations}

Revue française de référence sur les dynamiques

migratoires

$1293 \mid 2011$

L'immigration dans les musées

\title{
Communautés immigrantes, institution culturelle et espace politique
}

Le succès du Musée de l'immigration de Melbourne en Australie

\section{Ilham Boumankhar}

\section{(2) OpenEdition \\ Journals}

Édition électronique

URL : http://journals.openedition.org/hommesmigrations/503

DOI : 10.4000/hommesmigrations.503

ISSN : 2262-3353

Éditeur

Musée national de l'histoire de l'immigration

Édition imprimée

Date de publication : 1 septembre 2011

Pagination : 50-61

ISSN : $1142-852 X$

\section{Référence électronique}

Ilham Boumankhar, "Communautés immigrantes, institution culturelle et espace politique », Hommes \& migrations [En ligne], 1293 | 2011, mis en ligne le 31 décembre 2013, consulté le 22 avril 2019. URL : http://journals.openedition.org/hommesmigrations/503; DOI : 10.4000/hommesmigrations.503 


\title{
Communautés immigrantes, institution culturelle et espace politique
}

Le succès du Musée de l'immigration de Melbourne en Australie

\author{
Par llham Boumankhar, \\ doctorante à l'université Paris-I-Panthéon-Sorbonne et allocataire de recherche \\ au CRICC (Centre de recherche Images, Cultures et Cognitions) $)^{(1)}$
}

L'immigration en Australie revêt de multiples facettes. Inscrit dans une société australienne multiculturelle, le Musée de

l'immigration à Melbourne retrace en particulier la généalogie du peuplement de l'État de Victoria. En assumant les tensions entre l'histoire des colons et le sort réservé au peuple aborigène, ce musée met en scène une identité australienne en pleine évolution. Le fonctionnement de cette institution illustre la façon dont une société construite autour de l'immigration met en scène sa diversité. 
L'Australie est un pays qui constitue à lui seul un petit continent. Elle fait à la fois partie du Commonwealth et elle est, à ce titre, une ancienne colonie britannique de peuplement, mais c'est aussi un territoire isolé en Asie-Pacifique. Cette double appartenance politique et géographique fait de la question de l'immigration un enjeu social et culturel qui est renforcé par une histoire coloniale singulière. Il n'est donc pas étonnant que l'un des plus anciens musées dédiés à l'immigration soit l'Immigration Museum de Melbourne, situé dans l'État de Victoria. Ce musée a été crée à l'initiative du gouvernement de l'État de Victoria. Ce dernier, dès les années quatre-vingt, rassemble les acteurs qui feront partie du projet de création du musée et prend la décision de constituer une section spécifique dédiée à l'immigration mais qui reste rattachée au Museum Victoria ${ }^{(2)}$. Il s'agit d'expliciter le rôle social du Musée de l'immigration dans un contexte de politique multiculturelle et de dialogue intercommunautaire.

\section{De la discrimination raciale au multiculturalisme}

Le Musée de l'immigration de Melbourne a ouvert ses portes en 1998, dans un ancien bureau des douanes, lieu symbolique de contrôle de l'immigration et d'enregistrement des nouveaux arrivants. Son ouverture revêt une dimension socioculturelle importante dans un État qui est "l'un des plus culturellement variés d'Australie. Presque un quart de ses habitants est né à l'étranger, et 43,5\%, quand ils ne viennent pas eux-mêmes de l'étranger, ont au moins un parent qui n'est pas né sur le sol australien. La population provient de plus de 200 pays différents, parle plus de 180 langues ou dialectes et adhère à plus de 110 confessions religieuses ${ }^{(3)}$ ". L'Australie rassemble donc dans un même territoire un ensemble de communautés multi-ethniques et multiculturelles faisant de l'immigration un concept d'une grande complexité contextuelle.

Pourtant, si l'Australie pratique aujourd'hui une politique multiculturelle, celle-ci n'a été mise en place que depuis 1972, date à laquelle a été abrogée une politique d'immigration basée sur des critères de discrimination raciale et d'exclusion. En effet, si la Terra Australis est, tout d'abord, une destination carcérale, elle devient rapidement une colonie anglaise supplémentaire dans la conquête franco-britannique. La Grande-Bretagne et l'Irlande constituent les deux sources principales de peuplement pendant plus d'un siècle et demi, puisque la politique d'immigration se focalise alors sur la création d'une société idéale homogène et blanche.

Par la suite, d'autres pays européens viendront alimenter l'immigration de peuplement, avec des critères d'entrée sur le territoire qui s'appuient sur l'ethnicité des arrivants, 
conformément à la White Policy. "Pratiquée aujourd'hui, une telle politique susciterait à coup sûr réprobation et même indignation ${ }^{(4)}$."

Ce sont justement l'opinion internationale, les échanges interuniversitaires et la réalité géographique qui ont conduit le pays à réviser sa politique d'immigration de manière progressive. Lorsque le parti travailliste (le Labour Party) gagne les élections en 1972 , il abroge la White Policy et applique une politique calquée sur la diversité sociale ${ }^{(5)}$, mais qui permet aussi, implicitement, la reconnaissance des droits du peuple aborigène. Le multiculturalisme est une politique qui ne s'applique pas seulement à l'immigration mais aussi à l'ensemble des Australiens. Au-delà de la diversité culturelle et ethnique de l'Australie, il s'agit d'un ensemble de mesures gouvernementales qui répondent à la variété culturelle de la société australienne. La création du Musée de l'immigration participe au bon fonctionnement de cette politique multiculturaliste. La muséalisation de l'immigration permet de dégager de nouveaux espaces de discussion autour des cultures issues de l'immigration et d'exposer des objets offerts ou prêtés par des immigrants afin de raconter leur histoire. Le musée devient alors "un acteur social ancré dans un territoire, doté d'une légitimité et garantissant les contenus diffusés et un cadre spatio-temporel de la citoyenneté( ${ }^{\prime()}$ '. Le cas de l'Australie montre clairement que le musée est un outil politique chargé de défendre la politique multiculturelle du gouvernement et d'entretenir un dialogue permanent avec les communautés vivant dans l'État de Victoria. Une interaction sociale nécessaire, puisque cette politique a pour objectif de favoriser l'insertion des minorités ethniques au sein de la majorité, afin de créer " un corps social qui sache conjuguer diversité et homogénéité, qui ne soit pas une simple mosaïque de races ou de religions, où les ethnies se côtoieraient sans véritablement se rencontrer et encore moins se comprendre ${ }^{(7)}$. Le musée inscrit ses expositions dans l'histoire collective et politique de l'immigration australienne tout en intégrant le parcours individuel de chaque Australien, grâce à une collaboration permanente entre les acteurs du musée et les communautés immigrantes.

\section{Le dispositif muséal de l'lmmigration Museum de Melbourne}

L'Immigration Museum de Melbourne a pour objectif de présenter et d'explorer l'histoire des immigrants arrivés dans l'État de Victoria ${ }^{(8)}$. Disposant aussi d'un centre de découverte (the Discovery Center), il permet aux visiteurs de partager leur histoire, mais aussi de consulter une base de données généalogiques sur les communautés immigrantes de l'État. Quant à l'exposition permanente, elle présente l'histoire de l'immigration depuis l'arrivée des colons, le sort réservé au peuple aborigène, les 
différentes politiques d'immigration, les grandes vagues historiques d'immigrants et l'intégration des nouveaux arrivants dans la société d'accueil, avec la volonté d'incarner chaque histoire. Divers objets sont montrés dans l'exposition permanente : outils de travail, souvenirs de famille, produits culturels, objets traditionnels, objets de décoration ou du quotidien comme des vêtements, des chaussures, des accessoires... Chaque objet est placé sous une vitrine qui marque les différentes vagues migratoires ainsi que la diversité des nouveaux arrivants et introduit le visiteur à ce que représente l'immigration.

Le premier thème de l'exposition permanente est intitulé "Leaving home" et présente au visiteur les différentes raisons qui ont poussé des hommes et des femmes, parfois des familles entières, à quitter leur pays d'origine pour venir s'installer dans l'État de Victoria. Cette exposition se compose à la fois de sons, d'objets, d'images mobiles et de vidéos projetées afin d'explorer les motivations poussant les individus à immigrer et aussi ce que ces individus ont amené avec eux. Les sons recréent l'ambiance des ports maritimes qui accueillaient les immigrants par milliers. Les objets sont ceux que les immigrants ont apportés avec eux, en commençant par leurs valises. Les images mobiles sont une série de photographies projetées rapidement (20 images par seconde) de sorte à créer un mouvement. On y voit des hommes, des femmes et des enfants, tous âges confondus, avancer en portant une valise. Les vidéos retracent la réalité des parcours migratoires des immigrants, oscillant entre immigration forcée (pour cause de guerre) et immigration choisie (le désir d'une meilleure vie). Les textes qui accompagnent chaque objet et chaque partie de l'exposition permanente soulignent les origines diverses de l'immigration australienne.

D'autres thèmes composent l'exposition permanente, comme "Immigrant stories and timeline", "Journeys of a lifetime", "Getting in", "Station Pier : gateway to a new life", et présentent différents objets dont des outils témoignant du métier des nouveaux arrivants, des vêtements et costumes de traditions provenant de leurs pays d'origine, divers documents administratifs retraçant leurs voyages, depuis leur départ jusqu'à leur arrivé à Victoria.

\section{Faire vivre le processus migratoire}

Les différents motifs poussant les gens à immigrer en Australie sont mis en interrogation dès l'entrée du musée et s'accompagnent de différents types d'objets pour illustrer chaque période historique, telle que la mise en vitrine d'une boîte de la CroixRouge pour rappeler les conflits internationaux ou un fusil de la Seconde Guerre 
mondiale pour la guerre. Tout un étage du musée est consacré au port de Victoria, "Station Pier", lieu symbolique du passage de tous les immigrants. Une partie importante de l'exposition est consacrée à la politique d'immigration appliquée en Australie, avec un historique des mesures gouvernementales en fonction de la politique d'immigration, présentées à travers divers documents administratifs qui témoignent des différentes périodes d'application des mesures (passeports, pièces d'identité, et documents de voyages...).

On distingue quatre sections dans l'histoire de la politique d'immigration de l'Australie. La première entre 1840 et 1900, qui correspond à l'Australie en tant que second Empire britannique, puisque les colons venaient majoritairement de GrandeBretagne. La deuxième se situe entre 1901 et 1945 et correspond à l'application de l'Immigration Restriction Act, loi régissant l'accès au territoire australien selon des critères ethniques. La troisième période, entre 1946 et 1972, correspond à l'assouplissement de la politique d'immigration après la Seconde Guerre mondiale, ce qui permet des échanges avec des pays voisins, principalement d'Asie et du MoyenOrient. La quatrième période, entre 1973 et 2006, couvre la politique multiculturelle de l'Australie où la question de l'identité nationale reste toujours d'actualité. Cette dernière partie insiste sur la politique d'ouverture culturelle et ethnique de la société australienne, qui ne fige pas le concept d'identité dans une définition précise, mais s'adapte à la diversité des individus qui la composent. 


\section{Une participation active au questionnement identitaire}

Ainsi, dans la section "Immigration et identité nationale", l'histoire migratoire qu'a connue l'Australie pose diverses questions d'ordre sociétal liées à la définition de l'identité nationale: Quel genre de société voulons-nous? L'Australie est-elle simplement un avant-poste sud de la culture britannique ? ou son identité est-elle liée à l'Asie et au Pacifique? Y a-t-il un "type" d'identité australienne? La possibilité même qu'il existe un "type" d'identité dénie-t-elle la diversité de notre société ? Comment l'identité aborigène s'inscrit-elle dans l'idée de l'Australie en tant que nation d'immigrants? Est-ce que les différentes cultures peuvent préserver leur identité tout en participant à une "identité nationale"? Et le musée de préciser que la politique d'immigration fluctue selon "l'augmentation de la population, les besoins en main-d'ceuvre, les réponses aux besoins humanitaires mondiaux, qui ont été tempérés par des préférences de nationalité et de culture particulières ${ }^{(9) "}$. Le musée prend une position constructiviste en insistant sur l'ouverture à la diversité et en invitant les visiteurs à remettre en question des notions comme l'identité, la nation, l'immigration, la culture, la race ou l'ethnicité. Ce sont les histoires individuelles des arrivants, les difficultés administratives qu'ils ont rencontrées pour s'installer en Australie, les obstacles qu'ils ont dû surmonter pour s'intégrer dans la société australienne, ainsi que les différents modes d'adaptation au niveau professionnel, social et culturel qui sont explorés. Plusieurs installations sont aussi mises en place avec la volonté de faire vivre l'expérience des

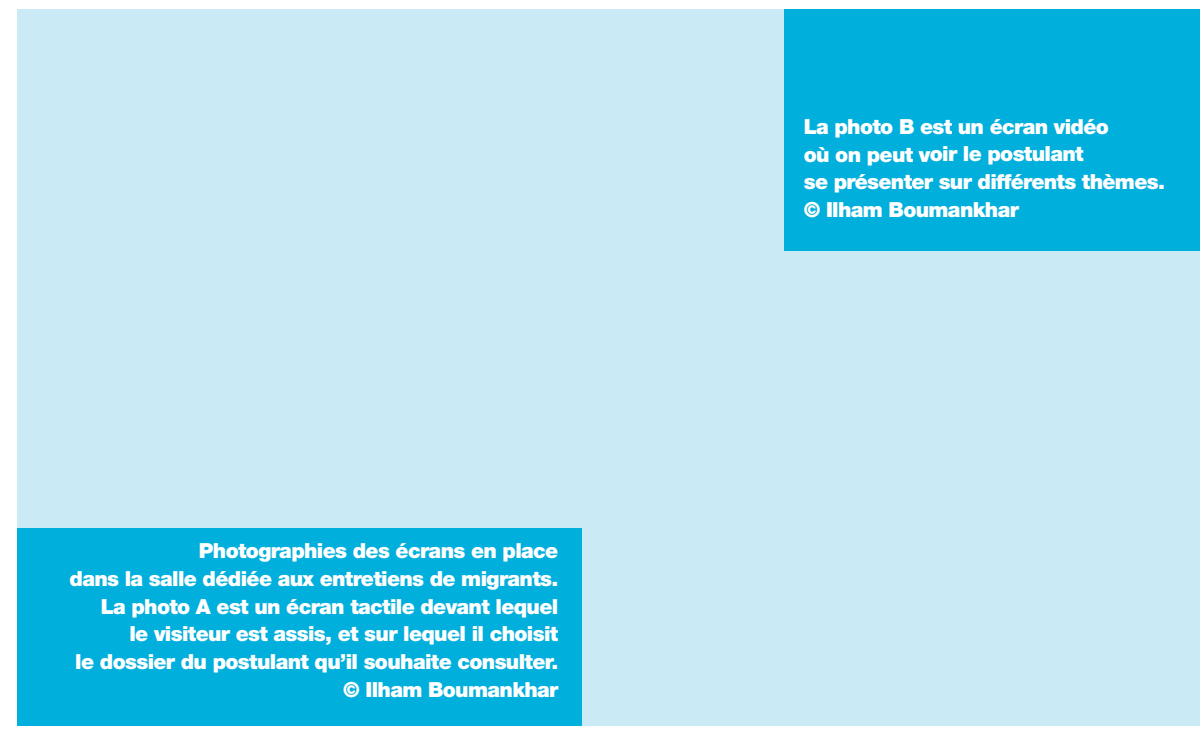


immigrants aux visiteurs, tel que le test de la dictée, qui servait à filtrer les immigrants en fonction de leur origine ethnique. Ainsi, le nouvel arrivant devait se faire dicter un texte dans une langue européenne et se voyait refuser l'entrée sur le territoire en cas d'échec. De même, une salle dédiée aux enretiens des migrants équipée d'écrans interactifs permet la simulation d'un interrogatoire entre un agent de contrôle de l'immigration et différents postulants au visa d'entrée. Le visiteur occupe la place de l'agent de contrôle et dispose d'une fiche détaillée qu'il doit compléter, alors qu'à l'écran un immigrant présente les raisons pour lesquelles il souhaite séjourner en Australie. À charge pour le visiteur de décider, via un écran tactile, si le candidat peut obtenir son visa d'entrée en Australie.

L'ambition du musée est de faire comprendre aux visiteurs les différents critères d'évaluation des agents de l'immigration durant les différentes périodes qui ont marqué les changements majeurs de la politique d'immigration australienne. La mise en exposition est conçue pour que chaque visiteur se sente impliqué par la question de l'immigration. En témoigne la "galerie des communautés" qui permet aux visiteurs de découvrir des objets, des photographies, des souvenirs ou des histoires personnelles. Maria Tence, directrice des expositions sur le thème des communautés du Museum Victoria, travaille en collaboration avec les représentants des communautés, les associations ou les familles appartenant à la communauté présentée. En moyenne trois sont présentées chaque l'année et, souvent, le choix est lié aux difficultés de compréhension rencontrées par rapport à leur histoire ou leurs pratiques culturelles. Lannée 2009-2010 a été consacrée à celles dont la situation dans leur pays reste tendue, voire parfois sans issue, comme les communautés palestinienne, kurde et timoraise. Le rôle de la galerie des communautés est de maintenir le dialogue avec les communautés vivant dans l'État de Victoria mais aussi d'établir des échanges entre elles. Enfin, les festivals culturels dédiés aux communautés ont pour but de favoriser ces échanges.

\section{Le musée comme espace de dialogue social}

Des entretiens réalisés avec les cadres de l'Immigration Museum de Melbourne ont permis de comprendre leur travail dans un musée qui traite d'une thématique identitaire, sociale et diversifiée. Padmini Sebastian, directrice de l'Immigration Museum de Melbourne ${ }^{(10)}$, souligne le rôle civique de ce musée, dont l'objectif est de représenter et de refléter l'État de Victoria sous l'éclairage de l'immigration. Il est ouvert depuis un peu plus de dix ans et "aucune ingérence de la part du 
gouvernement n'a jusqu'ici été notée", contrairement au Musée national australien. Le gouvernement était en effet intervenu à l'occasion de la mise en place d'une galerie consacrée à l'art aborigène en 2010.

Le musée doit toutefois se conformer aux politiques et aux procédures du gouvernement dans la gestion des ressources financières, tout en respectant le code de déontologie des musées. En terme de contenu, le musée est libre de traiter comme il l'entend les questions liées à l'immigration. Il arrive que le contenu diffusé corresponde aux questions gouvernementales et que cela devienne sujet à des contentieux, mais l'objectif est de pacifier les questions liées à l'immigration et d'offrir une zone d'échanges.

Le gouvernement australien, par sa politique de l'immigration, aura toujours pour ligne conductrice de déterminer, de démarquer ou de façonner l'identité de la nation australienne. Le rôle du musée n'est pas de juger cette ligne conductrice mais d'offrir

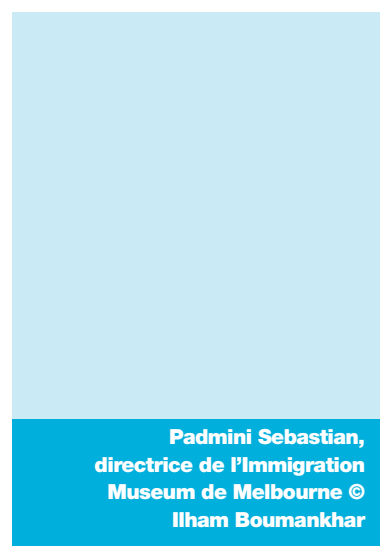
un espace de dialogue. Il s'agit d'amener les visiteurs mais aussi les citoyens à penser le musée non pas comme une institution culturelle mais comme un pôle social des questions de l'immigration. Les expositions à venir auront pour objectifs de présenter une nouvelle expérience interactive et immersive au visiteur, et un programme en ligne grâce auquel le visiteur pourra s'investir, partager, dans le respect des différences de chacun.

\section{Mettre en scène la diversité australienne}

Maria Tence ${ }^{(11)}$ a commencé à travailler avec les communautés il y a plus de douze ans, dans le cadre d'un projet d'exposition muséal en compagnie de Padmini Sebastian et Moya McFadzean, alors que le projet d'un musée de l'immigration à Melbourne se mettait en place. Depuis, son travail a beaucoup évolué. Si, au départ, il s'agissait d'établir un lien avec les différentes communautés et de leur expliquer l'intérêt d'une collaboration avec un musée civique et étatique, par la suite, il s'est agi davantage de négocier les différents modes de représentations et la diffusion des histoires individuelles et collectives. L'immigration en Australie présente une diversité intéressante. Les communautés sont d'installation plus ou moins ancienne, et si aujourd'hui la collecte 
des objets est plus simple, il n'en était pas de même à l'ouverture du musée. En effet, les immigrants venus en Australie après la Seconde Guerre mondiale n'étaient jamais entrés en contact avec une institution culturelle car la plupart venaient des zones rurales de l'Europe. Pour ces premiers immigrants, le musée était davantage un lieu où l'on entreposait des antiquités. Il a donc fallu entreprendre un travail pédagogique pour faire comprendre le rôle du musée dans la représentation des communautés qui a initié la communication entre le musée et les communautés. Une galerie des communautés à des fins exclusives de travail avec les communautés. En effet, pour que le musée acquière une légitimité à leurs yeux, il s'agissait à la fois de les intégrer à l'histoire sociale et contemporaine de l'immigration australienne et de leur réserver un espace spécifique. Au départ cette galerie s'appelait "Nexus Gallery". Le mot "nexus" signifie "le lien entre les membres d'un groupe ${ }^{(12) ",}$, en relation au partage de la même langue dans une communauté. Ce partage linguistique était davantage considéré par le musée comme un allié culturel que comme le facteur d'une insertion sociale plus facile. Le nom a changé en 2004 à la demande de plusieurs artistes qui voulaient faire de cet espace une entrée, un accès, une découverte artistiques. Or le Musée de l'immigration souhaitait un espace d'exposition réservé aux voix collectives et à l'histoire sociale. C'est donc devenu la "galerie des expositions communautaires".

Le rôle de Maria Tence est de faire comprendre aux communautés le bénéfice qu'elles ont à travailler avec le Musée de l'immigration. Le but est de garder une trace de leur arrivée en Australie en tant qu'immigrants mais aussi de transmettre leurs origines, leur histoire et leurs traditions culturelles. C'est en les amenant à raconter leur histoire que les individus appartenant à une communauté participent à l'histoire collective de la communauté. Cette histoire n'est pas celle de leur succès social en tant que nouveaux arrivants dans la société australienne mais celle des choix qui les ont poussés à venir en Australie, de leur bagage culturel et de leur contribution à la société australienne. Une société qui a elle aussi changé avec leur arrivée, modifiant son cadre législatif. Un équilibre intéressant que la galerie des expositions communautaires tente d'aborder et de refléter. Le but est d'offrir une vision globale de l'histoire de ces communautés anciennes ou récentes. 


\section{Accompagner le passage des témoignages aux objets}

Si la collection du Museum Victoria existe depuis 1854, celle du Musée de l'immigration est toute récente. Moya McFadzean ${ }^{(13)}$ a tout d'abord travaillé pour le Museum Victoria en 1995, en tant que conservatrice pour l'Australian Society and Technology Department. Parce qu'elle s'est spécialisée au cours de ses études dans l'histoire de l'immigration australienne et celle de l'État de Victoria en particulier, elle a été nommée conservatrice en chef des collections du musée de l'immigration de Melbourne. En tant que conservatrice nommée pour une nouvelle collection muséale, son travail a consisté à initier, tout d'abord, des liens avec les réseaux communautaires afin de développer un plan de collecte. Par la suite, il s'agissait de réaliser des collectes ciblées pour les expositions et de déterminer le profil du public du musée en termes de migrations liées à des activités culturelles. Elle définit son travail de collecte des objets comme un travail proactif, puisque le musée a la charge de créer et de compléter sa propre collection en partenariat avec les communautés de l'État de Victoria. L'objectif de cette collecte a été de couvrir un large éventail de cultures et de périodes historiques afin de refléter l'histoire des migrations de l'État de Victoria. L'accent a été mis sur la documentation des histoires individuelles et des migrations familiales à travers des objets personnels et des récits. Ce sont ces nombreuses histoires individuelles et familiales qui composent l'histoire globale de l'immigration australienne. La conservatrice commence à collecter des objets auprès des communautés. Au cours du processus de collecte, elle reçoit souvent les immigrants qui décident de partager leur histoire, afin de leur poser des questions précises relatives aux objets qu'ils prêtent ou qu'ils donnent. Le processus de collecte s'accompagne donc d'un travail de collaboration entre le propriétaire de l'objet et la conservatrice. Cet accompagnement est nécessaire car, souvent, la banalité des objets ou le fait qu'ils se fondent dans le paysage quotidien des propriétaires ont pour conséquence que ceux-ci ne sont pas

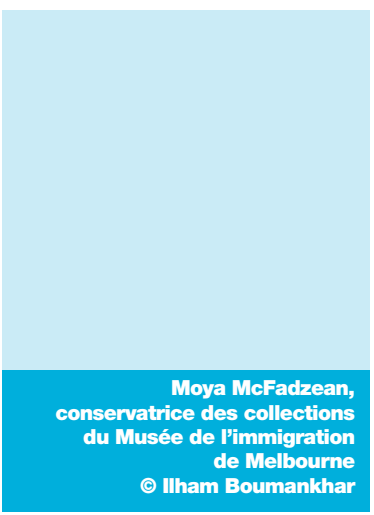
prolixes sur les détails des histoires qu'ils portent ou sur leurs usages passés. La conservatrice les aide à fournir un maximum de détails pour susciter un sentiment d'empathie chez le visiteur. Pour cela, Moya McFadzean réfléchit aux moyens techniques qui permettraient de relater les histoires personnelles attachées aux objets tout en les intégrant à l'histoire générale de l'immigration. 


\section{Conclusion}

Ces entretiens mettent en lumière les enjeux de la question et des représentations de l'immigration dans le contexte australien. Tout en étant le lieu de la diversité et du multiculturalisme, l'histoire sociale et politique de l'Australie présente des paradoxes et des exceptions qui démontrent comment l'immigration a participé au changement, aux tensions et au développement de l'expérience australienne moderne. Si le Musée de l'immigration de Melbourne a pour mission d'enregistrer et d'interpréter l'expérience des gens qui ont immigré vers l'État de Victoria et de promouvoir et de célébrer la diversité culturelle et l'identité australienne qui en résultent, cela nécessite une collaboration permanente avec les communautés et les acteurs sociaux qui participent au maintien de la cohésion sociale australienne, tout en modérant les événements politiques. Bien que l'immigration soit constitutive de l'histoire australienne, elle reste une dynamique sociale en mouvement permanent, qui demande au musée de s'engager dans un travail d'ajustement et de communication constant afin de favoriser les échanges interculturels. L'immigration produit une multiplicité de schémas constitutifs de l'identité des individus et de la diversité des réalités sociales. Les parcours migratoires sont tout aussi diversifiés, entre les immigrants ayant fui les guerres, les génocides, la famine, et ceux attirés par la réalité économique de l'Australie et en quête d'une nouvelle vie. Ce sont autant d'expériences de l'immigration qui se décline aussi à travers les générations, les différences ethniques (dans une Australie qui a été gouvernée par une politique blanche pendant plus d'un siècle), les pratiques culturelles (dans un pays dont le principe fondateur du multiculturalisme est la préservation des origines et des pratiques culturelles), ce qui se transmet à travers les langues, les religions, l'appartenance communautaire, etc. Même si le paysage social de ce pays rend le fait migratoire courant, l'histoire politique et sociale de l'Australie oblige le musée à traiter des questions de l'immigration avec beaucoup de précautions, afin de faciliter la transmission entre les générations ainsi que les rencontres entre les migrants et les populations d'accueil, à travers le récit de leur histoire personnelle. 


\section{BHbliographie}

- David Dutton, One of Us. A Century of Australian Citizenship, Sydney, UNSW Press, 2002.

- James Jupp, "Immigration and national identity : multiculturalism", in Geoff Stokes (dir.), The Politics of Identity in Australia, Cambridge, Cambridge University Press, 1997, pp. 132-144.

- James Jupp, From White Australia to Woomera. The Story of Australian Immigration, Cambridge, Cambridge University Press, Second Edition, 2007.

- Xavier Pons, Le Multiculturalisme en Australie, Paris, L'Harmattan, 1996.

- Xavier Pons, Les Mots de l'Australie, Toulouse, Presses universitaires du Mirail, 2005.

- Éric Richards, Destination Australia, Sydney, University of New South Wales Press, 2008.

- Alexandra Sauvage, "Between law and science : Australian museums dealing with aboriginal critique", in Pierre Lagayette (dir.), Rencontres australiennes. Regards croisés sur l'identité d'un peuple et d'une nation, Paris, Presses de l'université Paris-Sorbonne, 2008.

- Padmini Sebastian, "Mobiliser les communautés et transmettre leurs histoires : le rôle du musée de l'Immigration dans l'une des villes les plus multiculturelles du monde", in Museum International, n² 233/234, vol. 59, n 1/2, UNESCO, 2007.

- Geneviève Vidal, Contribution à l'étude de l'interactivité. Les usages du multimédia de musée. Bordeaux, Presses universitaires de Bordeaux, 2006.

- Keith Windschuttle, "How not to run a museum? People's history at the postmodern museum", in The Sydney Line, september 2001.

\section{Notes}

1. Ilham Boumankhar mène une recherche depuis 2007, pour sa thèse intitulée : "Muséographier l'immigration. Enquête sur la réception des objets exposés au musée (les cas de la Cité nationale de l'histoire de l'immigration à Paris, en France et de l'Immigration Museum à Melbourne, en Australie)."

2. Le musée de Victoria comprend le Melbourne Museum, l'Immigration Museum, le Scienceworks et le Royal Exhibition Building. Texte de présentation disponible sur le site officiel du musée: "Museum Victoria cares for the state's scientific and cultural collections, providing visitor access, activities and events at four distinct venues: Melbourne Museum, Immigration Museum, Scienceworks and the Royal Exhibition Building".

URL: http://museumvictoria.com.au/ (consulté le 8 Novembre 2009)

3. Padmini Sebastian, "Mobiliser les communautés et transmettre leurs histoires : le rôle du musée de l'Immigration dans l'une des villes les plus multiculturelles du monde", in Museum International, n 233/234, vol. 59, n 1/2, UNESCO, 2007, p. 157.

4. Xavier Pons, Le Multiculturalisme en Australie, Paris, L'Harmattan, 1996, p. 32.

5. James Jupp, From White Australia to Woomera. The Story of Australian immigration, Cambridge, Cambridge University Press, Second Edition, 2007.

6. Geneviève Vidal, Contribution à l'étude de l'interactivité. Les usages du multimédia de musée, Bordeaux, Presses universitaires de Bordeaux, 2006, p. 52.

7. Xavier Pons, op. cit., p. 24.

8. "The Immigration Museum explores the stories of real people from all over the world who have migrated to Victoria." Texte de présentation du musée disponible sur le site officiel du musée.

URL: http://museumvictoria.com.au/immigrationmuseum/about-us/ (consulté le 18 septembre 2010).

9. Texte de présentation: "Immigration and national identity" $\odot$ Immigration Museum de Melbourne (octobre 2009- mars 2010).

10. L'entretien s'est déroulé dans son bureau le 9 février 2010.

11. L'entretien s'est déroulé à la bibliothèque de recherche du musée, le 17 février 2010.

12. Nexus $[\mathrm{n}$ ks s] n. pl. nexus. Définition: 1. a means of connection between members of a group or things in a series; link; bond . 2. A connected group or series. Source URL: http://www.thefreedictionary.com/nexus (Consulté le 20 février 2010).

13. L'entretien s'est déroulé le 23 février 2010 au Swan House de l'Immigration Museum. 